\title{
Immunological subtypes in breast cancer are prognostic for invasive ductal but not for invasive lobular breast carcinoma
}

C C Engels ${ }^{1,4}$, D B Y Fontein ${ }^{1,4}$, P J K Kuppen ${ }^{1}$, E M de Kruijf ${ }^{1}$, V T H B M Smit ${ }^{2}$, J W R Nortier ${ }^{3}$, G J Liefers ${ }^{1}$, C J H van de Velde ${ }^{1}$ and E Bastiaannet ${ }^{\star, 1}$

${ }^{1}$ Department of Surgery, Leiden University Medical Center, Leiden, The Netherlands; ${ }^{2}$ Department of pathology, Leiden University Medical Center, Leiden, The Netherlands and ${ }^{3}$ Department of Medical Oncology, Leiden University Medical Center, Leiden, The Netherlands

Background: Classical patient and tumour characteristics are the benchmark of personalised breast cancer (BC) management. Recent evidence has demonstrated that immune and molecular profiling of $\mathrm{BC}$ may also play an important role. Despite evidence of differences between invasive ductal (IDC) and lobular (ILC) BC, they are infrequently accounted for when making treatment decisions for individual patients. The purpose of this study was to investigate the relevance of the tumour immune response in the major histological subtypes of BC. We also assessed the relationship between immune responses and molecular subtypes and their prognostic potential.

Methods: Immunostains were done for HLA-I, HLA-E, HLA-G, Tregs, NK cells and CTLs for the composition of the immune profiles and Ki67, EGFR, CK5/6, ER, PR and HER2 for molecular profiles in 714 breast cancer patients who underwent primary surgery.

Results: No significant association was found between IDC (90.6\%) and ILC $(9.4 \%)$ and tumour immune subtypes $(P=0.4)$ and molecular subtypes $(P=0.4)$. However, for the relapse-free period (RFP) tumour immune subtyping was prognostic $(P=0.002)$ in IDC, but not ILC. Contrary to ILC, IDC patients frequently expressed higher cleaved caspase-3 and Ki67, which was prognostic. Intermediate immune-susceptible IDC expressing high cleaved caspase-3 or Ki67 showed worse RFP than those with low expression (caspase-3: $P=0.004 ; \mathrm{Ki67}: P=0.002$ ); this was not seen for ILC or in high or low immune-susceptible tumour types for either IDC or ILC.

Conclusions: Tumour immune characteristics and host immune responses are prognostic in IDC, but not ILC. In addition, tumour immune profiles are only prognostic in Luminal A tumours.

Currently, breast cancer (BC) is the most commonly diagnosed cancer type and the leading cause of cancer-related death in the female population in the West (Jemal et al, 2011). Invasive ductal carcinoma (IDC) is by far the most common type of BC. The second largest group comprises invasive lobular carcinoma (ILC), and reports indicate that $10-15 \%$ of breast tumours are ILC (Arpino et al, 2004). Investigations into the differences between IDC and ILC have consistently shown that lobular carcinomas have a particular single-file growth pattern, tend to be larger, more often ER- and progesterone receptor (PR)-positive, and less aggressive than their ductal counterparts (Arpino et al, 2004; Mathieu et al, 2004). Nevertheless, these two types of BC are treated similarly with regard to systemic adjuvant therapy, which is based on tumour size, histological grade, hormone receptor status and human epidermal growth factor receptor 2 (HER2) status.

\footnotetext{
*Correspondence: Dr E Bastiaannet; E-mail: e.bastiaannet@|umc.nl

${ }^{4}$ These authors contributed equally to this work.
}

Revised 13 March 2014; accepted 15 May 2014; published online 17 June 2014

(c) 2014 Cancer Research UK. All rights reserved 0007-0920/14 
Gene expression studies have identified several distinct BC subtypes with marked differences in patient prognosis (Perou et al, 2000; Sorlie et al, 2001, 2003). This molecular classification proposed four different classes of breast tumours: Luminal A and B, basal-like and tumours overexpressing HER2. Luminal B tumours differ from Luminal A by a lower quantative content of hormone receptors. Basal-like tumours are triple-negative tumours and HER2-overexpressing tumours cluster near these basal-like tumours (Perou et al, 2000; Sorlie et al, 2001, 2003). Studies have shown that basal-like and HER2-overexpressing tumours have a more aggressive character, resulting in an unfavourable patient outcome compared to the Luminal tumour types.

With respect to over- and undertreatment, no optimal risk stratification exists for the allocation of the (individual) BC patient to the most appropriate therapeutic regimen. It is likely that the different gene expression profiles explain the observed survival differences seen in the BC population, even after controlling for tumour stage (Carey et al, 2006). However, there is also strong evidence that the $\mathrm{BC}$ host's adaptive immune system plays a crucial role in the control of tumour growth and progression (Zitvogel et al, 2006). On the other hand, breast tumour cells are able to adapt in order to escape the immune system and thus acquire characteristics to evade immunological recognition (Dunn et al, 2004). Several studies have attempted to elucidate this highly immunogenic disease by pointing out the great variety of immune reactions found in $\mathrm{BC}$.

Cytotoxic T-lymphocytes (CTLs) are capable of recognising tumour-associated antigens presented by classical human leukocyte antigen (HLA) class I on the tumour surface (Algarra et al, 2004). In order to avoid immune recognition from CTLs, cancer cells may lose expression of this classical HLA class I (Algarra et al, 2004). However, this makes them more prone to natural killer (NK) cell recognition (Wischhusen et al, 2007). Non-classical HLA class I molecules (HLA-E and HLA-G) play a crucial role in immune surveillance by NK cells. Expression of these molecules on the cell surface causes an inhibitory effect on NK-cell attack (Khong and Restifo, 2002; Marin et al, 2003; Wischhusen et al, 2007). Another tumour escape mechanism of immunosurveillance is attraction and induction of immunosuppressive regulatory $\mathrm{T}$ cells (Tregs) in the tumour microenvironment (Cerwenka et al, 2001). Together, these studies suggested that complex interactions take place between breast tumour cells and cells of the immune system. We recently reported on these complex interactions in a study on the immune subtypes of $\mathrm{BC}$, representing adaptive immune escape variants based on tumour-associated antigens (classical HLA class I and non-classical HLA-E and HLA-G) and tumour-infiltrating lymphocytes (CTLs, Tregs and NK cells) (de Kruijf et al, 2013). In this study, a clear association was observed between patient outcome and three tumour immune subtypes (low, intermediate and high immune subtypes) (Appendix Figure 1).

Our research group also demonstrated that the level of tumour cell proliferative and apoptotic signalling is an important factor in determining tumour development and thus predicting clinical outcome (Engels et al, 2013), and could thus very well add to the value of immunosubtypes in BC. Assuming that healthy tissue signifies a fine proliferative-apoptotic balance, we propose that tumour growth may be more accurately determined by the outcome of the balance between tumour cell proliferation (Ki67) on one side and apoptosis (cleaved caspase-3) on the other.

With the increasing ability of earlier diagnosis and subsequently the low relapse rate in early $\mathrm{BC}$ patients, in combination with the increasingly demanding nature of the contemporary patient population, the bar is raised for clinicians regarding optimal treatment (Tria, 2013). Therefore, individualised estimation of the true therapeutic benefit is of crucial importance, in order to avoid over- and undertreatment. Although the two major histological subtypes are frequently treated as similar entities, there are obvious differences in the tumour's biological and prognostic characteristics. The purpose of this study was to investigate the relevance of the host immune response, the apoptotic-proliferative interaction and molecular tumour types in the two major histological subtypes of $\mathrm{BC}$ and in different molecular tumour types.

\section{MATERIALS AND METHODS}

Patients and tumours. Non-metastatic BC patients who underwent primary surgical treatment at the Leiden University Medical Center (LUMC) between 1985 and 1996, with or without adjuvant systemic treatment, were included in the present cohort. Only patients with ILC or IDC were included in the study. Patients with bilateral tumours or an earlier history of cancer (other than basal cell carcinoma or cervical carcinoma in situ) were excluded. Data on age, histological type, tumour grade, TNM stage, ER, PR and HER2 were assembled. In addition, we collected information regarding local and systemic therapy and follow-up until locoregional and/or distant recurrence and/or death. All tumours were graded according to current pathological standards by a single BC pathologist (VTHBMS). Approval for the study was obtained from the LUMC Medical Ethics Committee. All samples were nonidentifiable and coded according to the national ethical guidelines (Code for Proper Secondary Use of Human Tissue, Dutch Federation of Medical Scientific Societies).

Immunohistochemistry. Formalin fixed paraffin-embedded tumour blocks of the primary tumour were collected at the pathology department. H\&E-stained sections with clear histopathological tumour representation were used for assembling tumour tissue microarray (TMA) paraffin blocks. From each donor breast tumour tissue block, three $0.6 \mathrm{~mm}^{2}$ tissue cores were punched from tumour areas and transferred into a recipient paraffin block using a custom-made precision instrument. Sections of $4 \mu \mathrm{m}$ were cut from FFPE tumour TMA material. Tissue sections were deparaffinised and rehydrated. Immunohistochemical staining was performed according to previously described standard protocols (de Kruijf et al, 2010b). As previously described, sections were incubated overnight with anti-Ki67 (mouse antihuman, M7240 Clone MIB-1: Dako Netherlands, Heverlee, Belgium), anti-cleaved caspase-3 (rabbit anti-human, anti-Asp175 \#9661: Cell Signaling, Leiden, The Netherlands) (Engels et al, 2013), anti-CD8 (mouse anti-human, ab 17147, clone 144B: Abcam, Cambridge, UK), anti-PEN5 (mouse anti-human, IM2354, clone 5H10.21.5: Beckman Coulter, Woerden, The Netherlands), mouse monoclonal anti-HCA2 and - $\mathrm{HC} 10$ directed against Classical HLA class I (anti-HLA-A and anti-HLA-B/C, respectively) and non-classical HLA class I molecules using mouse monoclonal antibodies against HLA-E (ab2216, clone MEM-E/02: Abcam) and HLA-G; ultimately, Treg infiltration was determined using anti-FoxP3 antibody (ab 20034, clone 236 A/E7: Abcam) with the predetermined optimal dilutions (de Kruijf et al, 2010a,b, 2013). For the molecular profiles additional staining was performed for EGFR (NCL-EGFR, Novocastra, Milton Keynes, UK) and CK5/6 (Clone D5/16 B4, Dako Netherlands). Immunohistochemical staining and quantification of ER, PGR and HER2 were performed previously. For each staining, all slides were stained simultaneously to avoid inter-assay variation. Negative controls were tissue sections that underwent the whole immunohistochemical staining with omission of the primary antibody.

Evaluation of the immunostaining. Expression of all markers was previously categorised as loss $v s$ expression for classical HLA class I; no expression vs expression for HLA-E and HLA-G; infiltration absent $v s$ infiltration present for Treg cells; and presence $v s$ absence of PEN5 (de Kruijf et al, 2010a,b, 2013). The absolute number of infiltrating CD8 + cells was microscopically 
assessed per $\mathrm{mm}^{2}$ and classified into two groups: two-thirds of patients with the lowest number of CD8 infiltration per $\mathrm{mm}^{2} v s$ one-third of patients with the highest number of CD8 infiltration per $\mathrm{mm}^{2}$ (de Kruijf et al, 2013). For cleaved caspase- 3 staining the mean expression grade of positively stained cells in the TMA was categorised into absent, low, intermediate and high scores. Cutoffs for low $v s$ high expression of Ki67, EGFR and CK5/6 were based on the median expression level (Engels et al, 2013).

Tumour immune subtypes. Tumour immune subtypes, representing tumour adaptive immune escape variants, were constructed with data from all known immunological variables of this patient cohort: classical HLA-I (HCA2 and HC10) and non-classical HLA-E and HLA-G expression, and Treg (FoxP3), CTL (CD8) and NK cell (PEN5) infiltration in the tumour material(de Kruijf et al, 2013). As described by de Kruijf et al, initially seven tumour immune subtypes were defined in ascending order from high immune susceptibility to low immune susceptibility. However, to facilitate clinical applicability the seven immune subtypes were condensed to a more simplified tumour immune subtype variable classification: high immune susceptibility, intermediate immune susceptibility and low immune susceptibility (de Kruijf et al, 2013). The latter subdivision was used in this experimental design.

Molecular subtypes. The IHC molecular profiles were previously developed by Carey et al (2006) and validated for inter-assay agreement using a gene expression assay. The IHC profile comprised the markers ER, PGR, HER2, Ki67, EGFR and CK5/6. The Luminal A profile was defined as $\mathrm{ER}+$ and/or PGR +, HER2 - and Ki67 - ; Luminal B as ER + and/or PGR + and $\mathrm{HER} 2+$ and/or Ki67 + ; ERBB2 as ER -, PGR - and HER2 + ; Basal-like as ER - , PGR - , HER2 - and EGFR + and/or CK5/ $6+$; and, lastly, the unclassified type as ER - , PGR - , HER2 - , EGFR - and CK5/6 -

Statistical analysis. Missing data were imputed (multiple imputation) using a model with IDC/ILC, grade, stage, age, follow-up and recurrence status, tumour immune subtypes, Ki67, caspase-3, molecular subtypes, ER, PR and HER2. With respect to multiple imputation, we generated 25 iterations and combined the estimates and standard errors using Rubin's Rules (micombine in STATA). Prior to running the model, checks were performed to test whether the data were missing at random. Multiple imputation by chained equations was used, which assumes a multivariate distribution exists without specifying its form. In STATA the ICE module was used to perform the multiple imputation. Univariable and multivariable binary logistic regression analyses were used to identify differences between IDC and ILC. All variables with a $P \leqslant 0.1$ in univariable analyses were entered into the multivariable model. Relapse-free period (RFP) was calculated using multivariable Cox proportional hazard models with any recurrence (locoregional recurrence and/or distant recurrence, whichever came first) as event, with the results stratified for IDC and ILC. Additional analyses were performed and stratified by age, tumour grade, tumour stage and nodal status. With respect to molecular subtypes, regression analyses were performed to assess proportional differences between molecular subtypes and immunological subtypes, caspase-3 and Ki67. In addition, Cox proportional hazard models were used to assess RFP in relation to immunological subtype, and stratified by molecular subtype. STATA/SE 12.0 version was used for all analyses.

\section{RESULTS}

Patient and tumour characteristics. Tumour material was available for $87 \%(704 / 822)$ of the patients. Median follow-up was 10 years (range $0.02-22$ years), and median age in this cohort was 58 years (range 23-96 years). Clinicopathological and treatment characteristics for the original and imputated cohorts are shown in Table 1.

IDC and ILC: differences in associations with clinicopathological parameters. No statistically significant difference was seen between IDC and ILC with regard to the association with tumour immune subtypes $(P=0.4)$ and molecular subtypes $(P=0.4)$. For the classical prognostic variables tumour grade $(P<0.001)$ and pathological tumour stage $(P=0.0002)$, a significant difference was seen between lobular and ductal breast tumour histology. ILC had significantly more grade II tumours and a higher pathological tumour stage. Both remained independent prognostic factors in the multivariate correction (grade: $P<0.001$, hazard ratio $(\mathrm{HR}): 12.6,95 \%$ confidence interval (CI): 3.5-44.8); pathological tumour stage: $P<0.0001$ (HR pT2: 2.3 (95\% CI: 1.1-4.8), HR pT3: 9.1 (95\% CI: 3.1-26.4), HR pT4: 10.3 (95\% CI: 3.0-35.5)). Also, compared to IDC, ILC showed a significantly lower expression pattern for both cleaved caspase-3 $\quad(P=0.0004, \quad$ HR low: $0.2 \quad(95 \%$ CI: $0.1-0.6)$, HR intermediate: 0.4 (95\% CI: $0.1-0.9)$, HR high: $0.1(95 \%$ CI: $0.01-0.4))$ and Ki67 ( $P=0.03$, HR: $0.4,95 \%$ CI: $0.2-0.9)$ following multivariable analyses.

Interaction with BC histology. An interaction test for RFP and histological subtype was performed to test for differences in effect between IDC and ILC. Results showed a significant effect modification for RFP for immune subtype $(P<0.001)$. In addition, similar results were observed with regard to active caspase- 3 $(P<0.001)$ and molecular subtype $(P=0.0005)$. With regard to Ki67, no effect modification was observed $(P=0.09)$. These findings indicate a possible influence of $\mathrm{BC}$ histology on the prognostic value of immune subtype, active caspase-3 and molecular subtype.

Relapse-free period in relation to IDC and ILC. Immunological profile was found to be prognostic for RFP in patients with IDC but not ILC, revealing a HR of 3.16 for low immune susceptibility compared to high immune-susceptible tumour types for IDC only $(P=0.002)$ (Table 2$)$. With regard to ILC a statistically significant association was only found in relation to immune subtype when stratified by tumour grade (grades I and II: $P<0.001$ and grade III: $P=0.01$ (data not shown).

For both high expression of apoptotic caspase-3 $(P=0.02, \mathrm{HR}$ 1.6, 95\% CI: 1.1-2.4) and high expression of proliferative Ki67 $(P=0.03$, HR $1.33,95 \%$ CI: $1.02-1.74)$ a significantly worse association was found with RFP in IDC, but not in ILC (Table 2). When stratified by pathological tumour stage, a significant association with RFP was only found for stage I and II tumours (high Ki67 HR: 1.37, 95\% CI 1.01-1.84, $P=0.04$ and high caspase3 HR: $1.85,95 \%$ CI: $1.21-2.81, P=0.0004)$ in IDC. With regard to IDC stage III and IV and ILC, no significant association was observed for Ki67 and caspase-3 (data not shown).

When caspase-3 and Ki67 were combined, a statistically significant association was observed in relation to RFP for IDC $(P=0.003)$, but not for ILC $(P=0.07)$ (data not shown).

The highest HR was seen for high caspase-3 expression combined with a high proliferative Ki67 rate (HR 2.0, 95\% CI: $1.2-3.3, P=0.003)$. When stratified by immune subtype, intermediate tumour immune phenotypes were significantly associated with caspase-3 $(P=0.004)$ and Ki67 $(P=0.002)$ expression regarding RFP. With increasing expression rate, both factors showed higher HRs (caspase-3 high: HR: 2.0, 95\% CI: 0.9-4.2 and Ki67 high: HR: 2.2, 95\% CI: 1.3-3.6). This was not observed in high or low tumour immune subtypes (data not shown).

Molecular subtypes: immune profiles and prognosis. There were no proportional differences between molecular subtypes and tumour immune subtypes $(P=0.6)$ (Table $3 \mathrm{~A})$. Luminal A tumours frequently did not express caspase-3, while high 


\begin{tabular}{|c|c|c|c|}
\hline & \multicolumn{2}{|c|}{ Original data set } & \multirow{2}{*}{\begin{tabular}{|c|}
$\begin{array}{c}\text { Multiple } \\
\text { imputations }\end{array}$ \\
$\%$ \\
\end{tabular}} \\
\hline & $N$ & $\%$ & \\
\hline \multicolumn{4}{|l|}{ Age } \\
\hline$<45$ & 137 & 19.2 & 19.2 \\
\hline $45-54$ & 175 & 24.5 & 24.5 \\
\hline $55-64$ & 157 & 22.0 & 22.0 \\
\hline $65+$ & 245 & 34.3 & 34.3 \\
\hline \multicolumn{4}{|l|}{ Year } \\
\hline 1985-1988 & 251 & 35.1 & 35.1 \\
\hline 1989-1992 & 232 & 32.5 & 32.5 \\
\hline 1993-1996 & 231 & 32.4 & 32.4 \\
\hline \multicolumn{4}{|l|}{ ER } \\
\hline Negative & 288 & 40.4 & 42.7 \\
\hline Positive & 393 & 55.0 & 57.3 \\
\hline Missing & 33 & 4.6 & \\
\hline \multicolumn{4}{|l|}{ PR } \\
\hline Negative & 316 & 44.3 & 48.0 \\
\hline Positive & 351 & 49.1 & 52.0 \\
\hline Missing & 47 & 6.6 & \\
\hline \multicolumn{4}{|l|}{ HER2 } \\
\hline No overexpression & 520 & 72.8 & 83.2 \\
\hline Overexpression & 59 & 8.3 & 16.8 \\
\hline Missing & 135 & 18.9 & \\
\hline \multicolumn{4}{|l|}{ Grade } \\
\hline Grade I & 116 & 16.3 & 16.8 \\
\hline Grade ॥ & 342 & 47.9 & 48.7 \\
\hline Grade III & 244 & 34.2 & 34.5 \\
\hline Missing & 12 & 1.7 & \\
\hline \multicolumn{4}{|l|}{ pT stage } \\
\hline pT1 & 289 & 40.5 & 41.4 \\
\hline pT2 & 328 & 45.9 & 47.2 \\
\hline рT3 & 44 & 6.2 & 6.5 \\
\hline pT4 & 33 & 4.6 & 4.9 \\
\hline Unknown & 20 & 2.8 & \\
\hline \multicolumn{4}{|l|}{ pN stage } \\
\hline Negative & 381 & 53.4 & 54.9 \\
\hline Positive & 313 & 43.8 & 45.1 \\
\hline Unknown & 20 & 2.8 & \\
\hline \multicolumn{4}{|c|}{ Histological subtype } \\
\hline IDC & 638 & 89.4 & 90.6 \\
\hline ILC & 66 & 9.2 & 9.4 \\
\hline Missing & 10 & 1.4 & \\
\hline \multicolumn{4}{|l|}{ Surgery } \\
\hline Mastectomy & 416 & 58.3 & 58.3 \\
\hline $\mathrm{BCS}$ & 298 & 41.7 & 41.7 \\
\hline \multicolumn{4}{|c|}{$\begin{array}{l}\text { Abbreviations: } B C S=\text { breast-conserving surgery; } E R=\text { oestrogen receptor; } H E R 2=\text { human } \\
\text { epidermal growth factor receptor } 2 ; I D C=\text { invasive ductal carcinoma; } I L C=\text { invasive lobular } \\
\text { carcinoma; } \mathrm{PN} \text { stage = pathological nodal stage; } \mathrm{PR}=\text { progesterone receptor; } \mathrm{PT} \text { stage = } \\
\text { pathological tumour stage. }\end{array}$} \\
\hline
\end{tabular}

Table 2. Association between breast cancer histological subtype and active caspase-3, Ki67, and immunological subtypes in relation to relapse-free period (all patients)

\begin{tabular}{|c|c|c|c|c|}
\hline \multirow[b]{2}{*}{ All patients } & \multicolumn{2}{|c|}{ Ductal breast cancer } & \multicolumn{2}{|c|}{ Lobular breast cancer } \\
\hline & $\begin{array}{c}\mathrm{HR}^{\mathrm{a}} \\
(95 \% \mathrm{Cl})\end{array}$ & $P$-value & $\begin{array}{c}\mathrm{HR}^{\mathrm{a}} \\
(95 \% \mathrm{Cl})\end{array}$ & $P$-value \\
\hline \multicolumn{5}{|c|}{ Immune subtypes } \\
\hline $\begin{array}{l}\text { High } \\
\text { Intermediate } \\
\text { Low }\end{array}$ & $\begin{array}{l}1 \text { (ref) } \\
1.95(1.09-3.48) \\
3.16(1.59-6.25)\end{array}$ & 0.002 & $\begin{array}{l}1 \text { (ref) } \\
2.10(0.51-8.73) \\
3.24(0.73-14.38)\end{array}$ & 0.3 \\
\hline \multicolumn{5}{|c|}{ Active caspase- 3} \\
\hline $\begin{array}{l}\text { Negative } \\
\text { Low } \\
\text { Intermediate } \\
\text { High }\end{array}$ & $\begin{array}{l}1 \text { (ref) } \\
1.04(0.72-1.52) \\
1.55(1.07-2.25) \\
1.58(1.06-2.36)\end{array}$ & 0.02 & $\begin{array}{l}1 \text { (ref) } \\
1.4(0.4-4.7) \\
3.8(0.8-17.3) \\
4.2(0.7-24.2)\end{array}$ & 0.2 \\
\hline \multicolumn{5}{|l|}{ Ki67 } \\
\hline $\begin{array}{l}\text { Low } \\
\text { High }\end{array}$ & $\begin{array}{c}1 \text { (ref) } \\
1.33(1.02-1.74) \\
\end{array}$ & 0.03 & $\begin{array}{c}1 \text { (ref) } \\
0.83(0.29-2.37) \\
\end{array}$ & 0.7 \\
\hline \multicolumn{5}{|c|}{$\begin{array}{l}\text { Abbreviations: } \mathrm{Cl}=\text { confidence interval; } \mathrm{HR}=\text { hazard ratio. } \\
{ }^{\mathrm{a}} \text { Adjusted for age, } \mathrm{pT} \text { and } \mathrm{pN} \text { (statistical interaction tests for histological subtype (IDC-ILC) } \\
\text { and immune subtypes: } P<0.001 ; \text { histological subtype (IDC-ILC) and active caspase- } 3 \text { : } \\
P<0.001 \text {; histological subtype (IDC-ILC) and Ki67: } P=0.09 \text {; histological subtype (IDC-ILC) } \\
\text { and molecular subtypes: } P=0.0005 \text { ). }\end{array}$} \\
\hline
\end{tabular}

expression was more prominent in Basal-like tumours $(P<0.001)$ (Table 3B). Basal-like tumours also expressed higher levels of Ki67 $(P<0.001)$ (Table 3C). As expected, luminal A tumours expressed low levels of Ki67, while luminal B tumours expressed high Ki67 levels (Table $3 \mathrm{C}$ ). Needless to say, immune profiles were strong prognostic indicators in Luminal A tumours, but not in Luminal B, HER2, or Basal-like tumours (Table 4). Luminal A tumours with low immune susceptibility showed a worse RFP than patients with high immune-susceptible tumours (high vs low: HR 3.9, 95\% CI: $1.5-10.1, P=0.006)$.

\section{DISCUSSION}

Previously, our study group reported that BC patients with particular immunological profiles were more susceptible to unfavourable outcomes, demonstrating that patients with low immune-susceptible tumours had a poorer prognosis when compared with intermediate or high immune-susceptible tumours (de Kruijf et al, 2013). Multiple research groups demonstrated that molecular profiling of $\mathrm{BC}$ also has important prognostic value (Perou et al, 2000; Sorlie et al, 2001). The current study focused on distinguishing the major histological subtypes to assess whether tumour immune and molecular profiles were of prognostic value. Our results show that tumour immune profiles are prognostic indicators in different histological subtypes of breast tumours.

IDC and ILC, by far, constitute the largest group of breast tumours, comprising up to $95 \%$ of all BCs. Although current treatment regimens do not distinguish between these histological subtypes, IDC and ILC are considered to be distinctive entities, and differentiating between these two subtypes may have a role in prognosis and the optimisation of $\mathrm{BC}$ treatment in addition to tumour size, histological grade, lymph node, ER/PR and HER2 status. The role of the immune response in cancer prognosis has been speculated on previously. Several studies have demonstrated a 
Table 3. Associations between molecular subtypes and immune subtypes (A), active caspase-3 (B), and Ki67 (C)

(A)

\begin{tabular}{|l|c|c|c|}
\hline Molecular subtypes $(\boldsymbol{P}=\mathbf{0 . 6})$ & Immune high & Immune intermediate & Immune low \\
\hline Unclassified & 14.0 & 74.5 & 11.4 \\
\hline Luminal A & 17.0 & 60.9 & 22.1 \\
\hline Luminal B & 19.7 & 61.9 & 18.4 \\
\hline HER2 & 16.0 & 58.4 & 25.6 \\
\hline Basal & 19.5 & 58.1 & 22.5 \\
\hline
\end{tabular}

(B)

\begin{tabular}{|l|c|c|c|c|}
\hline Molecular subtypes $(\boldsymbol{P}<\mathbf{0 . 0 0 1})$ & Caspase-3 negative & Caspase-3 low & Caspase-3 intermediate & Caspase-3 high \\
\hline Unclassified & 36.0 & 34.2 & 20.4 & 9.4 \\
\hline Luminal A & 37.8 & 35.5 & 19.0 & 8.7 \\
\hline Luminal B & 25.1 & 34.2 & 21.9 & 18.9 \\
\hline HER2 & 34.2 & 19.6 & 19.9 & 26.4 \\
\hline Basal & 18.9 & 25.2 & 24.7 & 31.2 \\
\hline
\end{tabular}

(C)

Molecular subtypes $(P<0.001)$

\begin{tabular}{|c|c|c|}
\hline Molecular subtypes $(P<0.001)$ & Ki67 low & Ki67 high \\
\hline Unclassified & 63.8 & 36.2 \\
\hline Luminal A & 92.6 & 7.4 \\
\hline Luminal B & 8.9 & 91.1 \\
\hline HER2 & 49.4 & 50.6 \\
\hline Basal & 34.4 & 65.6 \\
\hline
\end{tabular}

Abbreviation: HER2 = human epidermal growth factor receptor 2 .

Table 4. Associations of molecular and immunological subtypes with relapse-free period

\begin{tabular}{|l|l|c|c|}
\hline \multicolumn{2}{|c|}{} & \multicolumn{2}{c|}{$\begin{array}{c}\text { All histological } \\
\text { BC types }\end{array}$} \\
\hline \multirow{2}{*}{ Molecular subtypes } & Immune subtypes & $\begin{array}{c}\text { HR }^{\mathbf{a}} \\
(95 \% \mathrm{Cl})\end{array}$ & P-value \\
\hline Luminal A & High & $1($ ref) & 0.006 \\
& Intermediate & $1.8(0.8-4.4)$ & \\
& Low & $3.9(1.5-10.1)$ & \\
\hline Luminal B & High & $1($ ref $)$ & 0.4 \\
& Intermediate & $1.8(0.8-4.2)$ & \\
& Low & $2.0(0.7-5.9)$ & \\
\hline Basal-like & High & $1($ ref) & 0.1 \\
& Intermediate & $2.3(0.7-7.7)$ & \\
& Low & $3.8(1.2-12.5)$ & \\
\hline Abbreviation: BC=breast cancer. & & \\
a Adjusted for age, $\mathrm{PT}$ and pN; HER2 excluded due to too few numbers. & \\
\hline
\end{tabular}

correlation between single immune markers and patient outcome. It has been revealed that the presence of CD8 + lymphocytes, one of the most studied immune markers worldwide, in various types of cancer results in advantageous outcomes (Mahmoud et al, 2011). De Kruijf et al. demonstrated that the presence of classical HLA class I and high amounts of Treg infiltration affect prognosis in chemotherapy-treated BC patients only (de Kruijf et al, 2010b). In all probability, chemotherapy may help to selectively eliminate Treg, thus enabling CTLs to kill tumour cells that have retained HLA-I expression (de Kruijf et al, 2010b). The same group demonstrated that presence of non-classical HLA subtypes E and G was associated with a worse relapse-free period (de Kruijf et al, 2010a). This highly prognostic relation in BC was also seen when the immune markers were combined into immunological subtypes (de Kruijf et al, 2013). However, in none of the previous studies was the distinction made between IDC and ILC. Differences in histological subtype may evoke diverse responses on $\mathrm{BC}$ cells, thereby rendering one subtype more susceptible to the host immune response than another. In IDC patients, our analyses showed that low immune susceptibility as well as high caspase-3 and Ki67 expression were associated with a worse RFP, while this could not be demonstrated for ILC. These results also suggest that neither the apoptotic or proliferative marker, nor immune profiling applies to ILC, again suggesting that these tumours differ biologically from IDC.

With regard to molecular subtype, no correlation was observed between tumour immune subtype and molecular subtype. Based on previous studies, we know that tumours overexpressing HER2 and basal-like tumours generally present with more aggressive clinical characteristics than Luminal A and Luminal B tumours (Perou et al, 2000; Sorlie et al, 2001). Our results confirm that tumour aggressiveness, as established by molecular subtypes of $\mathrm{BC}$, is not dependent on a tumour's immunological profile. In addition, immunological profiling was found to be prognostic only for Luminal A tumours. Luminal A tumours make up the largest group of IDC. Therefore it is not surprising that these results show a similar prognostic association within the immune profiles. Jung et al (2010) proposed that ILC is frequently strongly ER+, HER2 - , and presents with low Ki67 expression, making it more likely to be characterised as a Luminal A molecular subtype. This finding may lead to the assumption that outcomes for molecular and histological subtypes are similar, but this was not confirmed in 
our analyses. This implies that a simple extrapolation cannot be made and that histological subtypes are presumably far more complex.

In this report we investigated the relationship of the clinical outcome of $\mathrm{BC}$ patients with immunological and histological profiles. Our results show that tumour immune biology differs greatly between IDC and ILC patients, confirming that ILC and IDC are completely different entities. Further studies are needed to validate these differences between IDC and ILC.

\section{ACKNOWLEDGEMENTS}

We thank the Dutch Cancer Society (KWF 2007-3968), and also Dr J Neefjes (Netherlands Cancer Institute, Amsterdam, The Netherlands) for providing anti-HLA-A and anti-HLA-B/C antibodies, and Professor Dr PJ van de Elsen (LUMC, Leiden, The Netherlands) for providing mouse monoclonal antibodies against HLA-G.

\section{CONFLICT OF INTEREST}

The authors declare no conflict of interest.

\section{REFERENCES}

Algarra I, Garcia-Lora A, Cabrera T, Ruiz-Cabello F, Garrido F (2004) The selection of tumor variants with altered expression of classical and nonclassical MHC class I molecules: implications for tumor immune escape. Cancer Immunol Immunother 53(10): 904-910.

Arpino G, Bardou VJ, Clark GM, Elledge RM (2004) Infiltrating lobular carcinoma of the breast: tumor characteristics and clinical outcome. Breast Cancer Res 6(3): R149-R156.

Carey LA, Perou CM, Livasy CA, Dressler LG, Cowan D, Conway K, Karaca G, Troester MA, Tse CK, Edmiston S, Deming SL, Geradts J, Cheang MC, Nielsen TO, Moorman PG, Earp HS, Millikan RC (2006) Race, breast cancer subtypes, and survival in the Carolina Breast Cancer Study. JAMA 295(21): 2492-2502.

Cerwenka A, Baron JL, Lanier LL (2001) Ectopic expression of retinoic acid early inducible-1 gene (RAE-1) permits natural killer cell-mediated rejection of a MHC class I-bearing tumor in vivo. Proc Natl Acad Sci USA 98(20): 11521-11526.

de Kruijf EM, Engels CC, van de Water W, Bastiaannet E, Smit VT, van de Velde CJ, Liefers GJ, Kuppen PJ (2013) Tumor immune subtypes distinguish tumor subclasses with clinical implications in breast cancer patients. Breast Cancer Res Treat 142(2): 355-364.

de Kruijf EM, Sajet A, van Nes JG, Natanov R, Putter H, Smit VT, Liefers GJ, van den Elsen PJ, van de Velde CJ, Kuppen PJ (2010a) HLA-E and HLA-G expression in classical HLA class I-negative tumors is of prognostic value for clinical outcome of early breast cancer patients. J Immunol 185(12): 7452-7459.

de Kruijf EM, van Nes JG, Sajet A, Tummers QR, Putter H, Osanto S, Speetjens FM, Smit VT, Liefers GJ, van de Velde CJ, Kuppen PJ (2010b) The predictive value of HLA class I tumor cell expression and presence of intratumoral Tregs for chemotherapy in patients with early breast cancer. Clin Cancer Res 16(4): 1272-1280.

Dunn GP, Old LJ, Schreiber RD (2004) The three Es of cancer immunoediting. Annu Rev Immunol 22: 329-360.

Engels CC, Ruberta F, de Kruijf EM, van Pelt GW, Smit VT, Liefers GJ, Matsushima T, Shibayama M, Ishihara H, van de Velde CJ, Kuppen PJ (2013) The prognostic value of apoptotic and proliferative markers in breast cancer. Breast Cancer Res Treat 142(2): 323-339.

Jemal A, Bray F, Center MM, Ferlay J, Ward E, Forman D (2011) Global cancer statistics. CA Cancer I Clin 61(2): 69-90.

Jung SY, Jeong J, Shin SH, Kwon Y, Kim EA, Ko KL, Shin KH, Lee KS, Park IH, Lee S, Kim SW, Kang HS, Ro J (2010) The invasive lobular carcinoma as a prototype luminal A breast cancer: a retrospective cohort study. BMC Cancer 10: 664.

Khong HT, Restifo NP (2002) Natural selection of tumor variants in the generation of 'tumor escape' phenotypes. Nat Immunol 3(11): 999-1005.

Mahmoud SM, Paish EC, Powe DG, Macmillan RD, Grainge MJ, Lee AH, Ellis IO, Green AR (2011) Tumor-infiltrating CD8 + lymphocytes predict clinical outcome in breast cancer. J Clin Oncol 29(15): 1949-1955.

Marin R, Ruiz-Cabello F, Pedrinaci S, Mendez R, Jimenez P, Geraghty DE, Garrido F (2003) Analysis of HLA-E expression in human tumors. Immunogenetics 54(11): 767-775.

Mathieu MC, Rouzier R, Llombart-Cussac A, Sideris L, Koscielny S, Travagli JP, Contesso G, Delaloge S, Spielmann M (2004) The poor responsiveness of infiltrating lobular breast carcinomas to neoadjuvant chemotherapy can be explained by their biological profile. Eur J Cancer 40(3): 342-351.

Perou CM, Sorlie T, Eisen MB, van de Rijn M, Jeffrey SS, Rees CA, Pollack JR, Ross DT, Johnsen H, Akslen LA, Fluge O, Pergamenschikov A, Williams C, Zhu SX, Lonning PE, Borresen-Dale AL, Brown PO, Botstein D (2000) Molecular portraits of human breast tumours. Nature 406(6797): 747-752.

Sorlie T, Perou CM, Tibshirani R, Aas T, Geisler S, Johnsen H, Hastie T, Eisen MB, van de Rijn M, Jeffrey SS, Thorsen T, Quist H, Matese JC, Brown PO, Botstein D, Lonning PE, Borresen-Dale AL (2001) Gene expression patterns of breast carcinomas distinguish tumor subclasses with clinical implications. Proc Natl Acad Sci USA 98(19): 10869-10874.

Sorlie T, Tibshirani R, Parker J, Hastie T, Marron JS, Nobel A, Deng S, Johnsen H, Pesich R, Geisler S, Demeter J, Perou CM, Lonning PE, Brown PO, Borresen-Dale AL, Botstein D (2003) Repeated observation of breast tumor subtypes in independent gene expression data sets. Proc Natl Acad Sci USA 100(14): 8418-8423.

Tria TM (2013) Breast cancer screening update. Am Fam Physician 87(4): 274-278.

Wischhusen J, Waschbisch A, Wiendl H (2007) Immune-refractory cancers and their little helpers-an extended role for immunetolerogenic MHC molecules HLA-G and HLA-E? Semin Cancer Biol 17(6): 459-468.

Zitvogel L, Tesniere A, Kroemer G (2006) Cancer despite immunosurveillance: immunoselection and immunosubversion. Nat Rev Immunol 6(10): 715-727.

This work is published under the standard license to publish agreement. After 12 months the work will become freely available and the license terms will switch to a Creative Commons AttributionNonCommercial-Share Alike 3.0 Unported License. 


\section{APPENDIX}

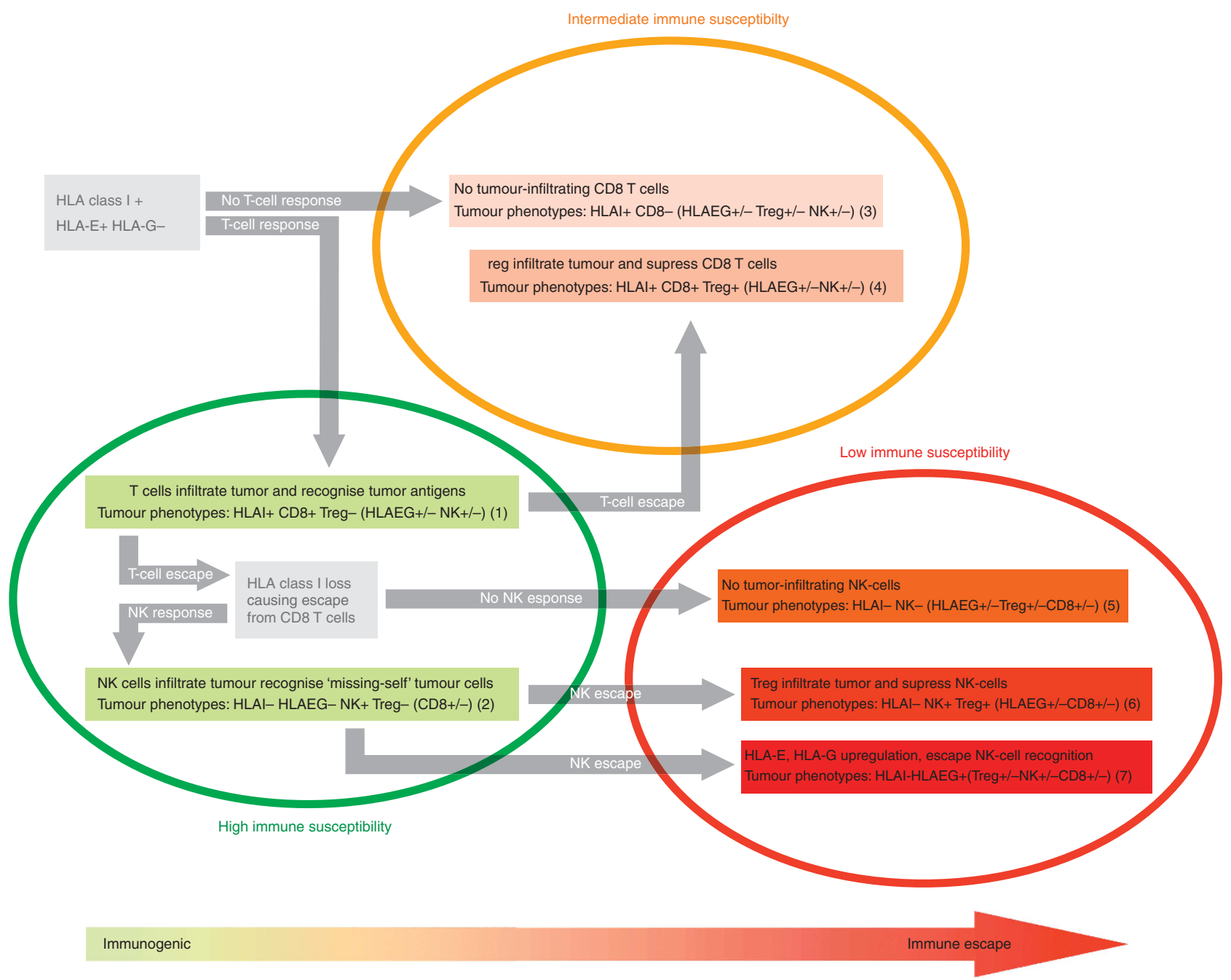

Appendix Figure 1. Tumour immune subtypes: showing a schematic overview of different stages of immune surveillance and tumour immune escape classified into seven immune subtypes, graded from 1 to 7 in ascending order from highly immunogenic and therefore high immune susceptibility (green) to high immune escape and low immune susceptibility (red), concerning combinations of CTL infiltration, NK-cell infiltration, Treg infiltration, classical HLA class I tumour expression, and HLA-EG tumour expression (de Kruijf et al, 2013). 